\title{
Erratum
}

\section{Tertiary Lymphoid Structures Associate with Tumour Stage in Urothelial Bladder Cancer}

Madhuri Koti, Amanda Shuo Xu, Kevin Yi Mi Ren, Kash Visram, Runhan Ren, David M. Berman and D. Robert Siemens

[Bladder Cancer 3(4), 2017, 259-267. DOI: 10.3233/BLC-170120]

https://content.iospress.com/articles/bladder-cancer/blc170120

On page 259, in the author listing, the author Amanda Shuo Xu was incorrectly listed as Amanda Shou Xu. 